\author{
Military Technical College \\ Kobry Elkobbah, \\ Cairo, Egypt \\ May 27-29,2008
}

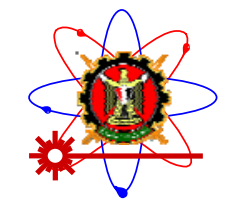

$4^{\text {th }}$ International Conference on Mathematics and Engineering Physics (ICMEP-4)

\title{
MODELING THE PRESSURE-VOLUME RELATION IN THE VENTRICLES
}

\author{
SHOUCRI ${ }^{*}$ R.M.
}

\begin{abstract}
The active force developed by the muscular fibres of the myocardium is represented as force per unit volume of the myocardium and included in the formalism describing the pressure-volume relation (PVR) and the end-systolic pressure-volume relation (ESPVR) in the left or right ventricle. Experimental methods to measure or to calculate the parameters describing PVR have often given inconsistent results. The objective of this study is to introduce some new mathematical relations describing the properties of PVR. Verifications based on published experimental data are given. Suggestions for future experimental work are indicated. The lack of adequate mathematical formalism to verify the consistency of experimental data is the main reason that prevents the application of PVR and ESPVR in routine clinical work.
\end{abstract}

\section{KEY WORDS}

Mechanics of ventricular contraction, clinical applications of pressure-volume relation,end-systolic pressure-volume relation, active force of the myocardium.

*Professor, Dpt. of Mathematics \& Computer Science, Royal Military College of Canada, Kingston, Ontario, Canada K7K 7B4. 


\section{NOMENCLATURE}

$a=$ inner radius of the myocardium.

$b=$ outer radius of the myocardium.

$h=b-a$ thickness of the myocardium.

$D_{r}=$ active radial force generated per unit volume of the myocardium.

$\bar{D} h=$ active radial pressure acting on unit area of the endocardium.

$(\bar{D} h)_{m}=$ maximum value of $\bar{D} h$.

$E=$ ventricular elastance, maximum value $E_{\text {max }}$.

$e_{a}=$ arterial elastance, maximum value $e_{a m}$.

$e_{d}=$ slope corresponding to $(\bar{D} h)$, maximum value $e_{d m}$.

$P=$ ventricular pressure.

$P_{o}=$ Pressure acting on the outer surface of the myocardium.

$V=$ ventricular volume.

$V_{e d}=$ end-diastolic volume.

$V_{e s}=$ end-systolic volume.

$V_{d}=$ intercept of the pressure-volume line with the volume axis.

$V_{d m}=$ value of $V_{d}$ when $E=E_{\text {max }}$.

$V_{\omega}=$ volume of the myocardium.

$S V=$ stroke volume.

$S W=$ stroke work

$C W=$ contraction work.

$P E=$ potential energy .

$S W R=$ stroke work reserve.

$T W=$ total area under ESPVR.

\section{INTRODUCTION}

Several interesting studies related to the pressure-volume relation (PVR) can be found in the literature [1-24], Many indexes used to assess the contractility of the myocardium are based on the study of PVR in the left or right ventricle of the heart. These include ejection fraction, stroke volume, stroke work, maximum ventricular elastance $\left(E_{\max }\right)$ or slope of the end-systolic pressure-volume relation (ESPVR), maximum arterial elastance $\left(e_{a m}\right)$. Possible adaptation of ESPVR to short-term or long-term change in systemic load condition has been discussed [7-9 ]. Different areas under ESPVR offer a sensitive index to assess the state of the myocardium $[7,10]$, and relations between these areas and oxygen consumption have been reported [11,12]. The ratio $E_{\max } / e_{a m}$ (maximum ventricular elastance/corresponding arterial elastance) has been used to express the ventriculo-arterial coupling [7-9, 13], this ratio can also be used to differentiate between normal, mildly or severely depressed physiological state of the heart as discussed in what follows [see also 7,15-19]. Experimental verification of this important result can be found in the literature [20-22]. Because of the need to measure the pressure in an invasive way by catheterization, it is important to note that these results are obtained by using invasive methods of measurement on animals or humans. Volume measurement however can de done in non-invasive way by ultrasounds or MRI for instance.

The main difficulty in implementing the preceding results in a non-invasive way for routine clinical work is the inconsistency in the calculation of the parameters describing PVR based on single-beat estimation when different experimental 
techniques are used [23]. Part of the difficulty may be experimental, but part is also due to the need of adequate mathematical formalism that can relate in a reliable way the parameters measured or calculated in order to verify the consistency of the results. In this study some mathematical relations are introduced for this purpose that relate the parameters describing PVR. Particular attention is given to the way the active force of the myocardium (also called isovolumic pressure) is included in the formalism describing PVR and the mechanics of left ventricular contraction. Based on data taken from the literature, experimental verification of the mathematical relations derived is given and suggestions for future experimental research in this field are indicated. Although the formalism discussed in what follows applies to both the right and left ventricle [24], we shall restrict our attention to applications related to the left ventricle.

\section{MATHEMATICAL FORMALISM}

Various authors $[25,26]$ have used a cylindrical model for the left ventricle. The left ventricle is represented as a thick-walled cylinder contracting symmetrically as shown in Fig. 1, a helical fibre in the myocardium is projected on the cross-section as a dotted circle. Because of the assumed symmetry of the contraction, a radial active force/unit volume of the myocardium $D_{r}$ is generated by the myocardium. The main originality of the mathematical formalism used is the inclusion of $D_{r}$ in the formalism describing PVR, as shown in what follows. $D_{r}$ will generate a radial pressure on the endocardium given by $\int_{a}^{b} D_{r} d r=\bar{D} h$, where $\bar{D}$ is an average value calculated by using the mean value theorem, $h=b-a$ is the thickness of the myocardium, $a=$ inner radius, $b=$ outer radius. In a quasi-static approximation (inertia and viscous forces neglected), the equilibrium of forces on the inner surface of the myocardium (endocardium) can be directly deduced from Fig. 1 and expressed in the form

$$
\bar{D} h-P=E\left(V_{e d}-V\right)
$$

Equ. 1 can be split into two equations

$$
\begin{aligned}
& P=E\left(V-V_{d}\right) \\
& \bar{D} h=E\left(V_{e d}-V_{d}\right)
\end{aligned}
$$

We have the following definitions that are shown in Fig. 1 and Fig. 2

$$
\begin{aligned}
& V=\text { ventricular volume } \\
& V_{e d}=\text { ventricular volume at end-diastole (when } d V / d t=0 \text { ) } \\
& P=\text { ventricular pressure } \\
& E=\text { ventricular elastance, slope of the pressure-volume line in Fig. } 2 \\
& V_{d}=\text { volume intercept of the pressure-volume line in Fig. } 2
\end{aligned}
$$

When $E$ reaches its maximum value $E_{\max }$ near end-systole as shown in Fig 2, we add a suffix $m$ to the previous equations as shown in the following equations 


$$
\begin{aligned}
& (\bar{D} h)_{m}-P_{m}=E_{\text {max }}\left(V_{e d}-V_{m}\right) \\
& P_{m}=E_{\text {max }}\left(V_{m}-V_{d m}\right) \\
& (\bar{D} h)_{m}=E_{\text {max }}\left(V_{e d}-V_{d m}\right)
\end{aligned}
$$

We have assumed the approximation $V_{m} \approx V_{e s}$ (end-systolic volume when $d V / d t \approx 0$ ) which will be used in our computation.

In the simplified diagram of Fig $2 V_{e d} d_{2} d_{1} V_{m}$ represents the pressure-volume loop in a normal ejection contraction, its area being equal to the stroke work $S W$. The line $d_{3} V_{d m}$ represents the ESPVR. The area $S W$ reaches its maximum value $(S W)_{\max }$ when the point $d_{1}$ coincides with $d_{5}$ (mid-point of the segment $d_{3} V_{d m}$ ). We can consequently distinguish between three different clinical cases as previously discussed in [7,15-19], normal physiological state of the ventricle corresponds to $E_{\text {max }} / e_{a m} \approx 2$ or $P_{m} /(\bar{D} h)_{m} \approx 1 / 3\left(d_{1}\right.$ below $d_{5}$ on the line $d_{3} V_{d m}$, this corresponds to maximum efficiency for oxygen consumption by the myocardium); mildly depressed state of the ventricle corresponds to $E_{\max } / e_{a m} \approx 1$ or $P_{m} /(\bar{D} h)_{m} \approx 1 / 2\left(d_{1}\right.$ and $d_{5}$ coincides, $S W$ reaches its maximum value $\left.(S W)_{\max }\right)$; severely depressed state of the ventricle corresponds to $E_{\max } / e_{a m} \approx<1$ or $P_{m} /(\bar{D} h)_{m}>1 / 2\left(d_{1}\right.$ above $\left.d_{5}\right)$. These theoretical results are experimentally verified in the work of Burkhoff and Sagawa [20], Asanoi et al [21] and De Tombe et al [22].

Notice that Equ (1) or Equ (3) can be looked at in two different ways:

a) If $(\bar{D} h)_{m}$ is held constant and $P_{m}$ and $V_{m}$ are varied, we obtain a straight line relationship like the line $d_{3} V_{d m}$ near end-systole, as if a balloon is inflated against a constant $(\bar{D} h)_{m}$.

b) If $\bar{D} h$ is is allowed to vary with $P$ and $V$, then we obtain the P-V loop $V_{e d} d_{2} d_{1} V_{m}$ shown in Fig. 2 as in a normal ejecting contraction. $\bar{D} h$ varies from $\mathrm{d}_{2}$ to $\mathrm{d}_{4}$ in Fig. 2 during a normal ejecting contraction.

Mathematical derivation of these results based on the theory of large elastic deformation can be found in [27, 28], and in the case of linear elasticity it can be found in $[29,30]$.

\section{RELATIONS BETWEEN SLOPES AND AREAS UNDER ESPVR}

Fig. 3 shows different areas under ESPVR $d_{3} V_{d m}$ that are obtained when the radial active force/unit area $(\bar{D} h)_{m}$ generated on the inner surface of the myocardium (endocardium) is included in the mathematical formalism describing PVR. The relative amplitude of these areas plays an important role in assessing the performance of the heart as a pump.

These areas are clearly shown in Fig. 3 from which we define the following:

$S W=$ area $V_{e d} d_{2} d_{1} V_{m}$, stroke work (or external work) delivered to the systemic circulation.

$C W=$ area $d_{1} d_{2} d_{3}$, apparent contraction work absorbed by the passive medium of the myocardium. 
$P E=$ area $V_{m} d_{1} V_{d m}$, potential energy apparently related to the internal metabolism in the myocardium.

$S W R=$ area $d_{1} d_{2} d_{6} d_{5}$, stroke work reserve (or external work reserve) $=(S W)_{\max } \quad-\quad S W$,

$E_{\text {max }}=$ maximum ventricular elastance, slope of ESPVR $d_{3} V_{d m}$.

$e_{a m}=$ arterial elastance corresponding to $E_{\max }$.

$e_{d m}=$ slope related to $(\bar{D} h)_{m}$

The main difficulty in implementing ESPVR for routine clinical work is how to measure or calculate $E_{\max }$ and $V_{d m}$ in a way that the different areas under ESPVR can be calculated and applied in clinical work, the areas under ESPVR are sensitive indexes that reflect the state of the myocardium. Quantities like $V_{m} \approx V_{e s}$ or the stoke volume $S V \approx V_{e d}-V_{m}$ can be calculated in a non-invasive way by MRI or ultrasounds, $P_{m}$ can be measured in invasive way by catheterization. The main problem is how one can estimate $E_{\max }, V_{d m}$ or equivalently $e_{d m}$ from parameters that can be measured. In what follows we give some relations that relate the areas under ESPVR to $e_{d m}$ and suggest some ways to estimate $e_{d m}$ and $V_{d m}$.

From Figs 2 and 3 we have the relations

$$
\begin{aligned}
& P_{m}=e_{a m} S V \\
& (\bar{D} h)_{m}=e_{d m} S V
\end{aligned}
$$

as well as the relation

$$
\frac{e_{d m}}{e_{a m}}=\frac{V_{e d}-V_{d m}}{V_{m}-V_{d m}}
$$

from which $V_{d m}$ can be calculated if $e_{d m} / e_{a m}$ is known and vice versa. Some simple mathematical calculations give the following expressions for the areas under ESPVR

$$
\begin{aligned}
& \frac{S W}{T W}=2\left(\frac{e_{d m}-e_{a m}}{e_{d m}}\right)\left(\frac{e_{a m}}{e_{d m}}\right) \\
& \frac{C W}{T W}=\left(\frac{e_{d m}-e_{a m}}{e_{d m}}\right)^{2} \\
& \frac{P E}{T W}=\left(\frac{e_{a m}}{e_{d m}}\right)^{2} \\
& P E \times C W=\frac{S W^{2}}{4}
\end{aligned}
$$

From these relations it is seen that the main problem to be solved in order to determine the areas and contours under ESPVR is to know how to estimate $e_{d m} / e_{a m}$ or equivalently $E_{\max }$ and $V_{d m}$, notice that $e_{a m}$ can be estimated from Equ. $5_{1}$. In order 
to estimate these parameters we give in what follows some experimental results based on data taken from the literature and we point out to some suggestions for future experimental work.

\section{EXPERIMENTAL APPLICATIONS}

We have taken some experimental data presented in Table 1 in [31] from experiments on 10 human subjects with no cardiovascular disease, as well as data given in [32,33] all based on experiments on dogs. Figs 4 and 5 show the apparent linear trend in the variation of $e_{d m}$ with respect to the variation of $e_{a m}$, further experimental investigation of this relation is needed. Another possible relation comes from Equ. 6 and exploits the fact that $e_{a m} / e_{d m}=\left(V_{m}-V_{d m}\right) /\left(V_{e d}-V_{d m}\right) \approx V_{m} V_{e d}$ since $V_{d m}$ is small, experimental results are shown in Fig. 6 . This relation would allow the calculation of $e_{d m}$, and consequently $(\bar{D} h)_{m}$, since $V_{m} / V_{e d}$ and $e_{a m}$ can be determined experimentally. A similar relation between $\left(V_{m}-V_{d m}+V_{\omega}\right) /\left(V_{e d}-V_{d m}+V_{\omega}\right)$ and $\left(V_{m}+\right.$ $\left.V_{\omega}\right) /\left(V_{e d}+V_{\omega}\right)$ is shown in Fig $7, V_{\omega}$ is the volume of the myocardium. Both $V_{m} / V_{e d}$ and $\left(V_{m}+V_{\omega}\right) /\left(V_{\text {ed }}+V_{\omega}\right)$ can be measured in a non-invasive way by using for instance echocardiography. Finally Fig. 8 presents two relations the apparent linearity of which suggests the need for further investigation that can allow to establish consistent relations between the parameters involved.

\section{CONCLUSIONS}

The main problem to be solved in order to apply PVR and ESPVR for routine clinical work is the difficulty in estimating the different parameters needed to describe these relations, this difficulty is increased when it comes to single-beat measurement. One of the reasons behind this difficulty is the lack of adequate theoretical formalism that can be used by experimentalists in order to verify the consistency of their experimental measurements. This study has presented some results that can be used for future experimental studies aimed at solving this problem. The references at the end of this study are not exhaustive and have been compiled in order to give to interested researchers a broad view of the subject, both from a theoretical and from an experimental point of view.

\section{REFERENCES}

1. Sagawa K, Maughan L, Suga H, Sunagawa K: Cardiac Contraction and the Pressure-Volume Relationship, Oxford University Press, New York and Oxford, 1988. 2. Bann J, Bachman K, Gibson D, eds: Ventricular function and pressure-volume analysis, the role of conductance in cardiology. Eur Heart $\mathrm{J}$ (suppl E) 13: 1-118, 1992.

3. Ingels NB Jr, Daughters GT, Baan J, Covell JW, Reneman RS, Yin FC-P: Systolic and diastolic function of the heart. IOS Press 1996.

4. Suga H: Paul Dudley White international lecture: cardiac performance as viewed through the pressure-volume window. Jpn Heart J 35: 263-280, 1993.

5. Kass DA, Kelly RP: Ventriculo-arterial coupling, concepts, assumptions and applications. Ann Biomed Eng 20: 41-62, 1992. 
6. Burkhoff D, Sugiura S, Yue DT, Sagawa K: Contractility dependent curvilinearity of end-systolic pressure-volume relations. Am J Physiol 252: H1218-H1227, 1987. 7. Shoucri RM: Possible clinical applications of the external work reserve of the myocardium. Jpn Heart J 35: 771-787, 1994.

8. Shoucri RM: Clinical application of end-systolic pressure-volume relation. Ann Biomed Eng 22: 212-217, 1994.

9. Shoucri RM: The active force of the myocardium. In: Computer Simulation in Biomedicine, Power $\mathrm{H}$ and Hart RT eds., Computational Mechanics Publications, Southampton UK and Boston USA, p. 19-26, 1995.

10. Crottogini AJ, Willshaw P, Barra JG, Armentano R, Cabrera Fisher EI, Pichel RH: Inconsistency of the slope and the volume intercept of the end-systolic pressurevolume relationship as individual indexes of inotropic state in conscious dogs, presentation of an index combining both variables. Circulation 76: 1115-1126, 1987. 11. Suga $H$ : Total mechanical energy of a ventricle model and cardiac oxygen consumption. Am J Physiol 236: H498-H505, 1979.

12. Shoucri RM: Theoretical study related to left ventricular energetics. Jpn Heart J 34: 403-417, 1993.

13. Little WC, Cheng CP: Left ventricular arterial coupling in conscious dogs. Am J Physiol 261: H70-H76, 1991.

14. Shoucri RM: Non-linear pressure-volume relation in left ventricle. Jpn Heart J 32, 337-346, 1991.

15. Shoucri RM: Ventriculo-arterial coupling and the areas under the end-systolic pressure-volume relation. Jpn Heart J 38:253-262, 1997.

16. Shoucri RM: The areas under ESPVR and the ventriculo-arterial coupling. In: Simulations in Biomedicine IV, Power H, Brebbia CA and Kenny J eds.,

Computational Mechanics Publications, Southampton UK and Boston USA, p. 47-56, 1997.

17. Shoucri RM: Studying the mechanics of left ventricular contraction. IEEE Eng Med Biol 17, p. 95-101, 1998.

18. Shoucri RM: A mathematical model for the contraction of the myocardium, $2^{\text {nd }}$ European Medical and Biological Eng. Conf. EMBEC02, IFMBE Proceedings, vol. 1, p.622-623 (4-8 DEC 2002, Vienna, Austria).

19. Shoucri RM: The end-systolic pressure-volume relation and its application to the study of the contractility of the cardiac muscle, Computers in Cardiology 33, 297300, 2006.

20. Burkhoff D, Sagawa K: Ventricular efficiency predicted by an analytical model. Am J Physiol 250: R1021-R1027, 1986.

21. Asanoi H, Sasayama S, Kamegama T: Ventriculo-arterial coupling in normal and failing heart in Humans. Circ Res 65: 483-493, 1989.

22. De Tombe PP, Jones S, Burkhoff D, Hunter WC, Kass DA: Ventricular stroke work and efficiency both remain nearly optimal despite altered vascular loading. Am J Physiol 264, H1817-1824, 1993.

23. Kjorstad KE, Korvald C, Myrmel T: Pressure-volume-based single-beat estimations cannot predict left ventricular contractility in vivo. Am J Physiol 282: H1739-H1750, 2002.

24. Shoucri RM: Pressure-volume relation in the right ventricle. J Biomed Eng 15: 167-169, 1993.

25. Humphrey JD and Yin FC: Constitutive relations and finite deformations of passive cardiac tissue II: stress analysis in the ventricle. Circ Res 65: 805-817, 1989. 26. Guccione JM, McCulloch AD and Waldman LK: Passive material properties of intact ventricular myocardium determined from a cylindrical model. ASME J Biomech Eng 113, 42-55, 1991. 
27. Shoucri RM: The pressure-volume relation and the mechanics of left ventricular contraction. Jpn Heart J 31, 713-729, 1991.

28. Shoucri RM: Theoretical study of pressure-volume relation in left ventricle. Am J physiol 260, H 282-H291, 1991.

29. Shoucri RM: Active and passive stresses in the myocardium. Am J Physiol 279, H2519-H2528, 2000.

30. Shoucri RM: Comparison between linear elasticity and large elastic deformation in the study of the contraction of the myocardium. In: Modelling in Medicine and Biology VII, Brebbia CA ed., WIT Press, Southampton UK and Boston USA, p. 3-14, 2007.

31. Borow KM, Newman A, Wynne J: Sensitivity of end-systolic pressure-dimension and pressure-volume to the inotropic state in humans. Circulation 65: 988-997, 1982. 32. Burns JW, Covell JW, Myers R, Ross J Jr: Comparison of directly measured left ventricular wall stress and stress calculated from geometric reference figures. Cir Res 28, 611-621, 1971.

33. Shoucri RM: The calculation of the intramyocardial stress. Tech \& Health Care 10, 11-22, 2002.

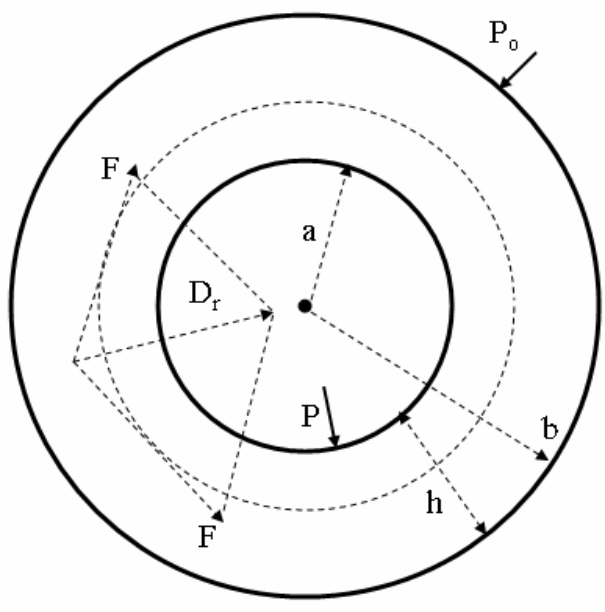

Fig 1: Cross-section of a thick-walled cylinder representing the left or right ventricle. The dotted circle represents the projection of a helical fibre on the cross-section. $D_{r}(r)$ is the radial active force/unit volume of the myocardium. $P$ is the ventricular pressure, $P_{o}$ is the outer pressure on the epicardium (neglected), $a=$ inner radius, $b=$ outer radius, $h=b-a=$ thickness of the myocardium.

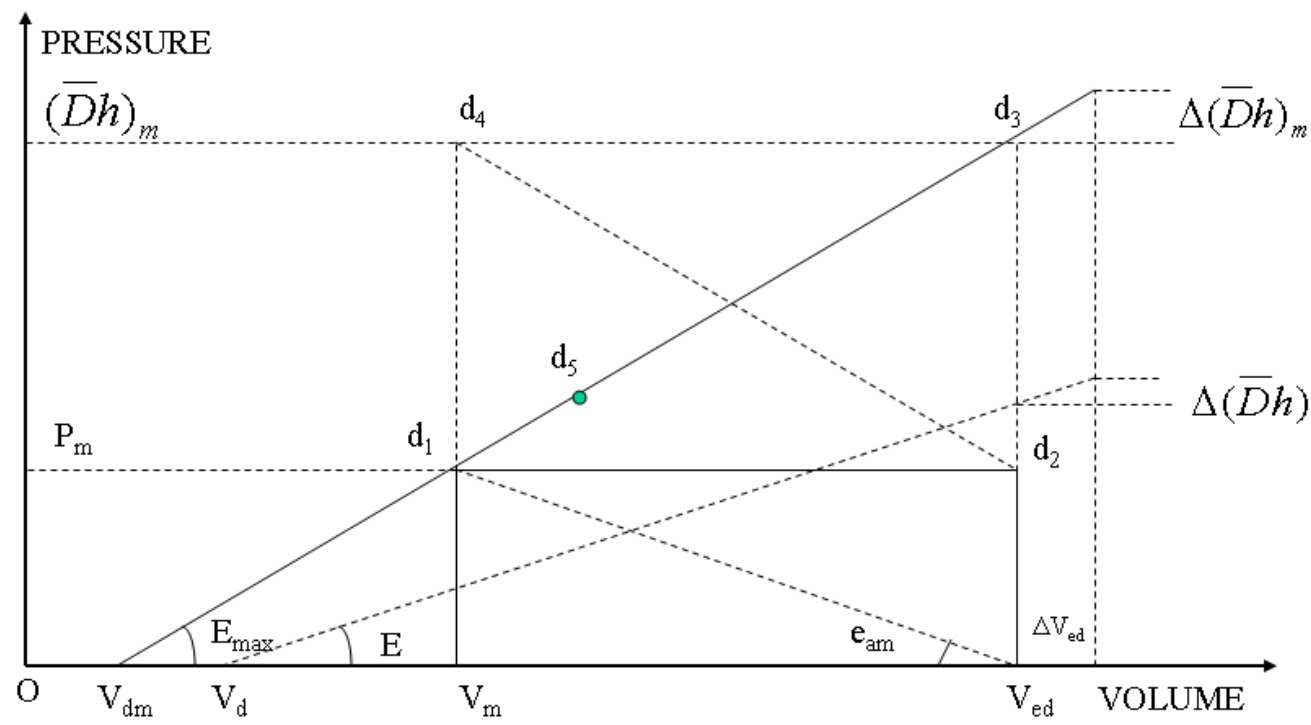

Fig. 2: Simplified drawing showing the pressure-volume relation (PVR) in the left or right ventricle. The end-systolic pressure-volume relation (ESPVR) is represented by 
the line $d_{3} V_{d m}$. PVR in a normal ejecting cycle is represented by the loop $V_{e d} d_{2} d_{1} V_{m}$ $(\bar{D} h)_{m}$ is the peak radial active force/unit area generated by the muscular fibres on the inner surface of the myocardium (peak isovolumic pressure). It is assumed that $V_{m} \approx V_{e s}$ (end-systolic volume when $\left.d V / d t=0\right)$. The changes $\Delta(\bar{D} h)$ and $\Delta(\bar{D} h)_{m}$ correspond to the change $\Delta V_{e d}$ in the end-diastolic volume according to the FrankStarling mechanism.

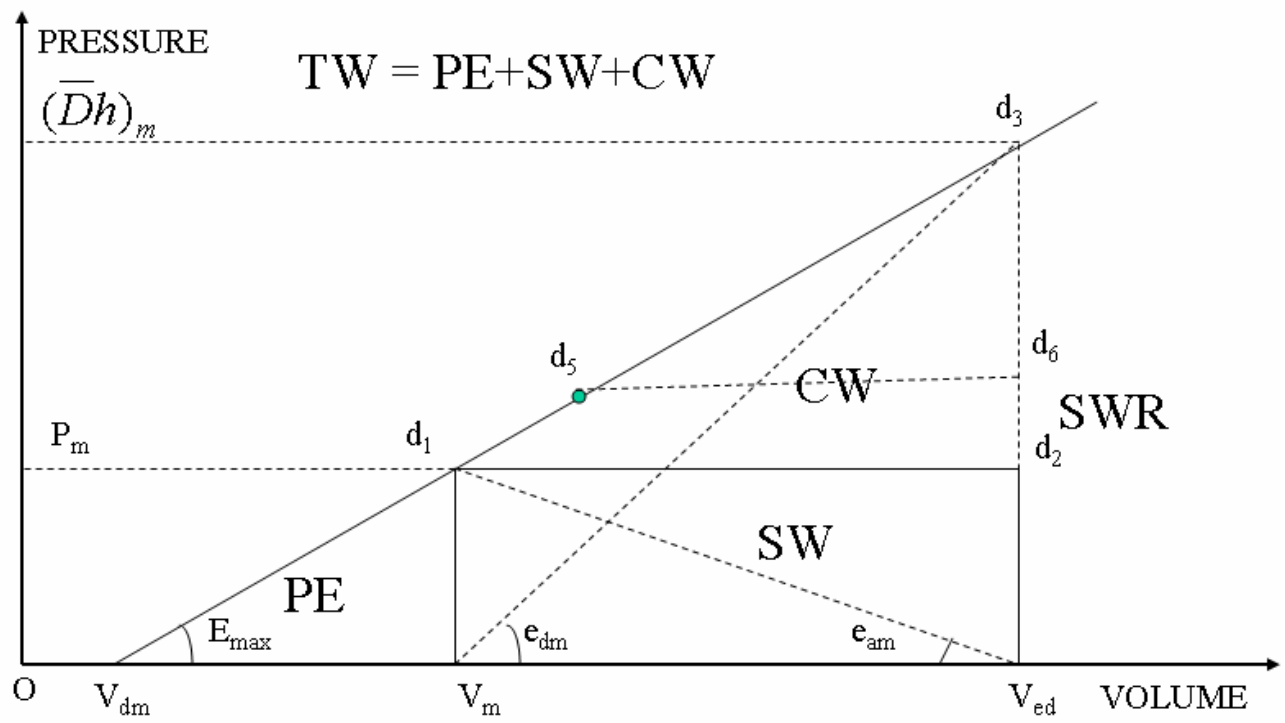

Fig. 3: Different areas under ESPVR $d_{3} V_{d m}$. $S W=$ stroke work area $V_{e d} d_{2} d_{1} V_{m} ; C W=$ contraction work area $d_{1} d_{2} d_{3} ; P E=$ potential work area $V_{m} d_{1} V_{d m} ; S W R=$ stroke work reserve area $d_{1} d_{2} d_{6} d_{5}, d_{5}$ is the mid-point of of the segment $d_{3} V_{d m} ; T W=$ total area under ESPVR. $E_{\max }=$ slope of ESPVR; $e_{a m}=$ corresponding arterial elastance; $e_{d m}=$ slope related to $(\bar{D} h)_{m}$.

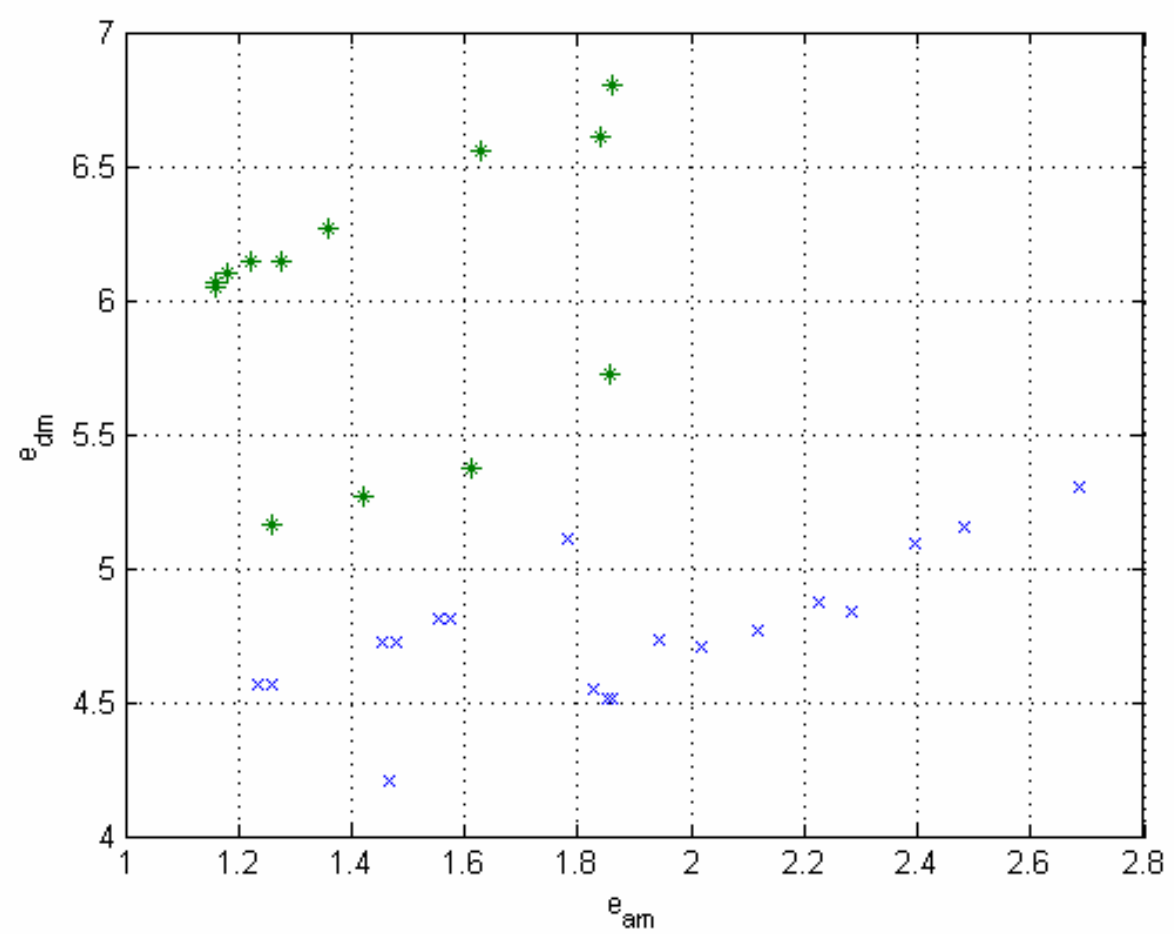


Fig. 4: Variation of $e_{d m}$ with $e_{a m}$ for four experiments based on data taken from Table 1 of Borow et al [31]. (x) control values, $\left({ }^{*}\right)$ corresponding data after injection of dobutamine. Notice the apparent linearity of the relation.

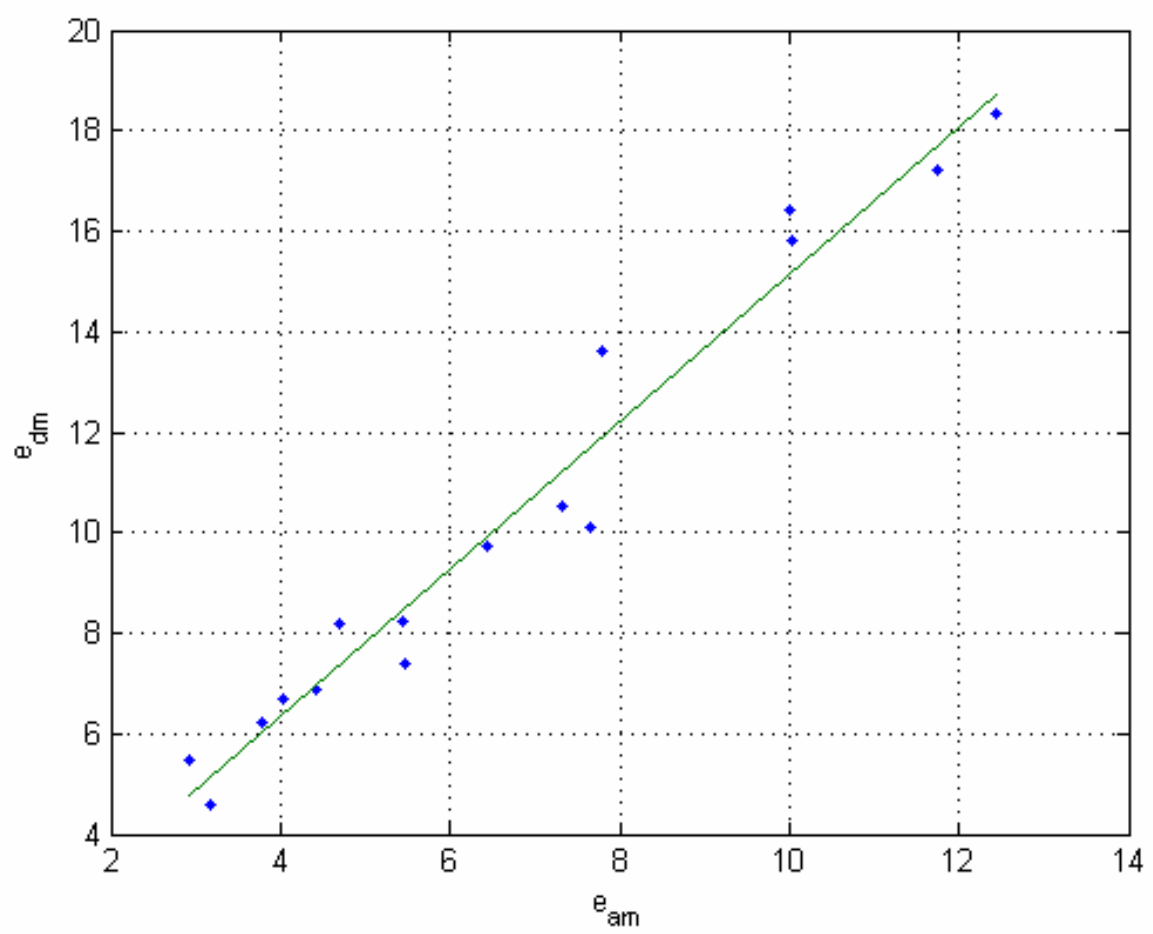

Fig. 5: Variation of $e_{p m}$ with $e_{a m}$ based on experimental data taken from Burns et al [32] and Shoucri [33]. Notice the apparent linearity of the relation. Least squares fit gives $y=1.4626 x+0.5227$, correlation factor $r=0.9816$. 


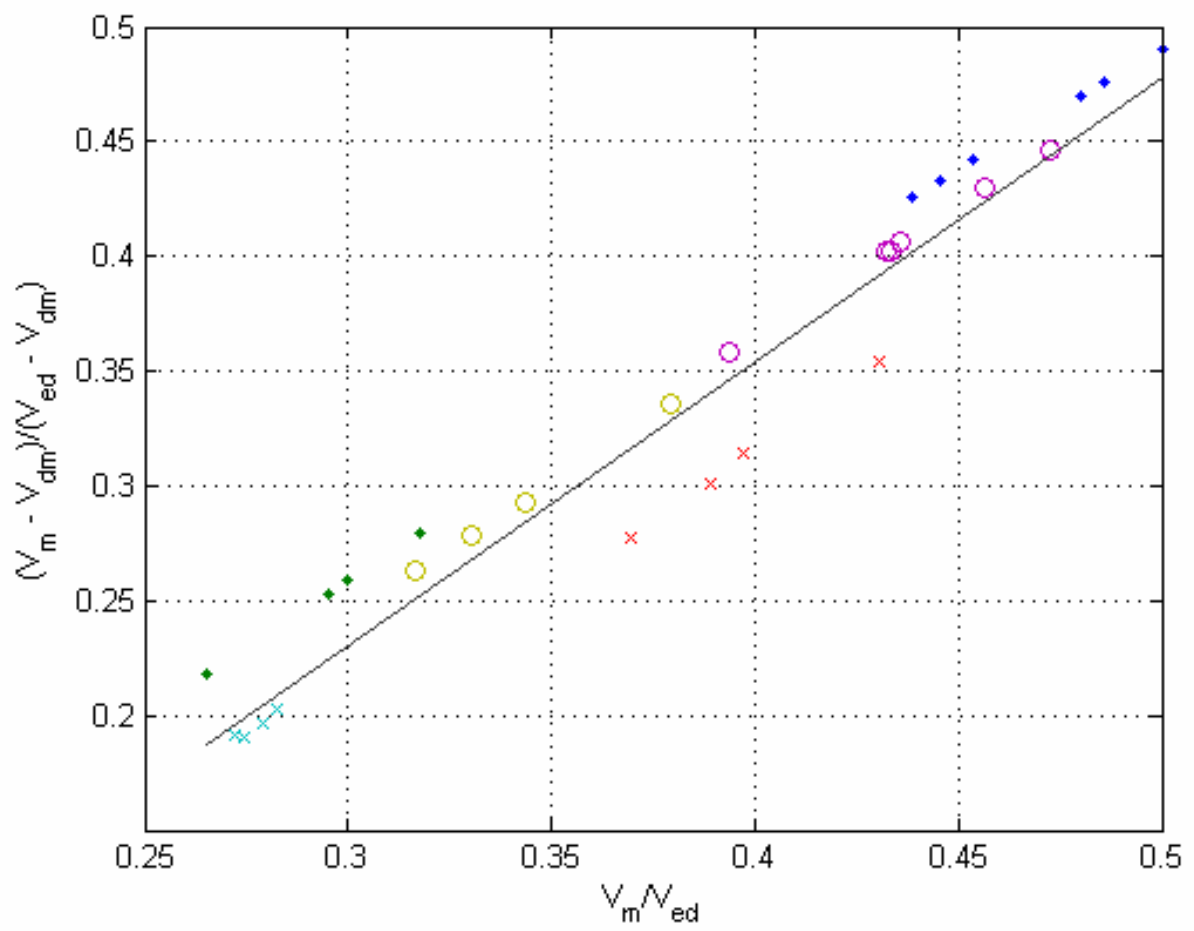

Fig. 6: Apparent linear relationship from which $y=\left(V_{m}-V_{d m}\right) /\left(V_{e d}-V_{d m}\right)$ can be determined from $x=V_{m} / V_{e d}$. Least squares fit gives $y=1.2377 x-0.1477$ with correlation coefficient $r=0.9686$. Data are taken from Table 1 of Borow et al [31].

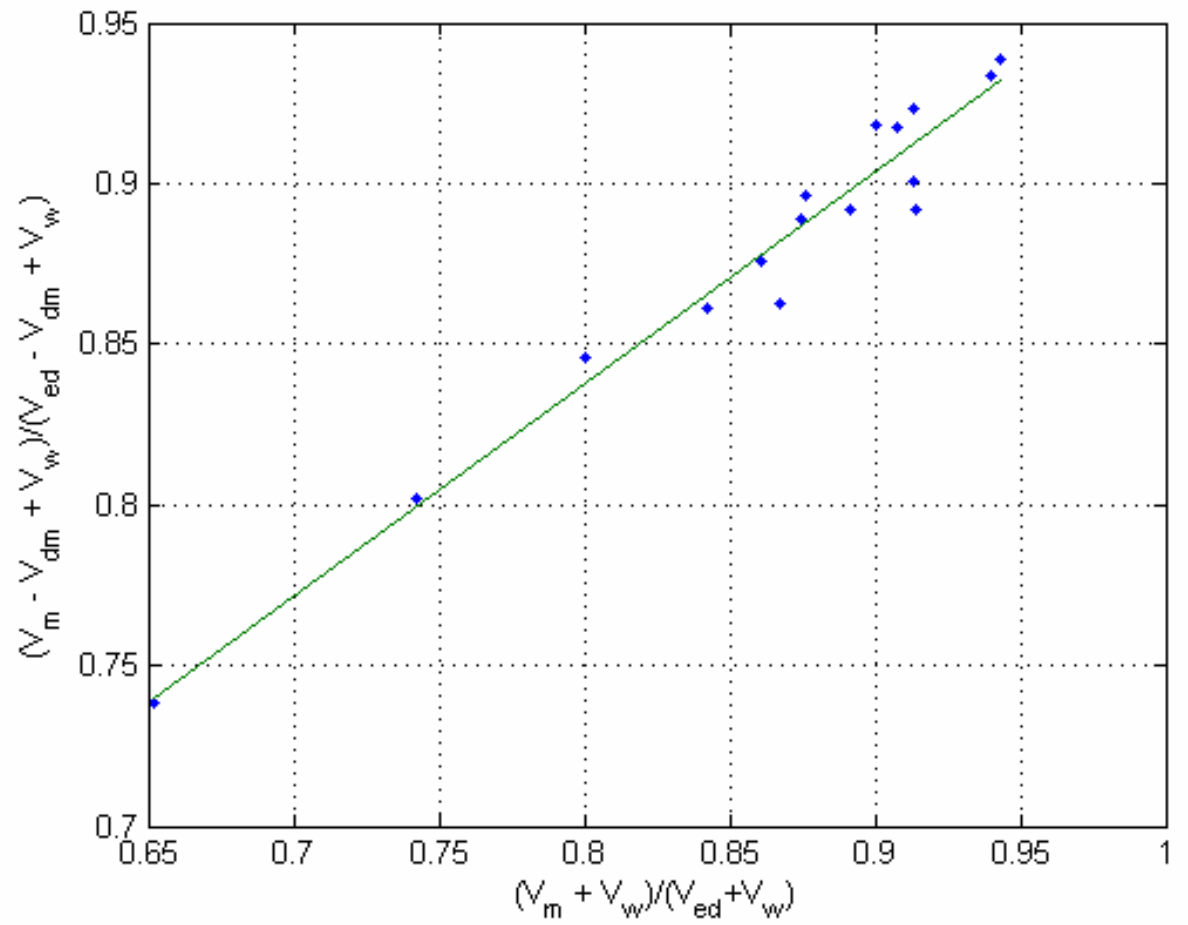

Fig. 7: Relationship between $y=\left(V_{m}-V_{d m}+V_{\omega}\right) /\left(V_{e d}-V_{d m}+V_{\omega}\right)$ and $x=\left(V_{m}+V_{\omega}\right) /\left(V_{e d}+V_{\omega}\right)$. Experimental data are taken from Burns et al [32] and Shoucri [33]. Least squares fit gives $y=0.661 x+0.3086$, correlation coefficient $r=$ 0.9798 . 

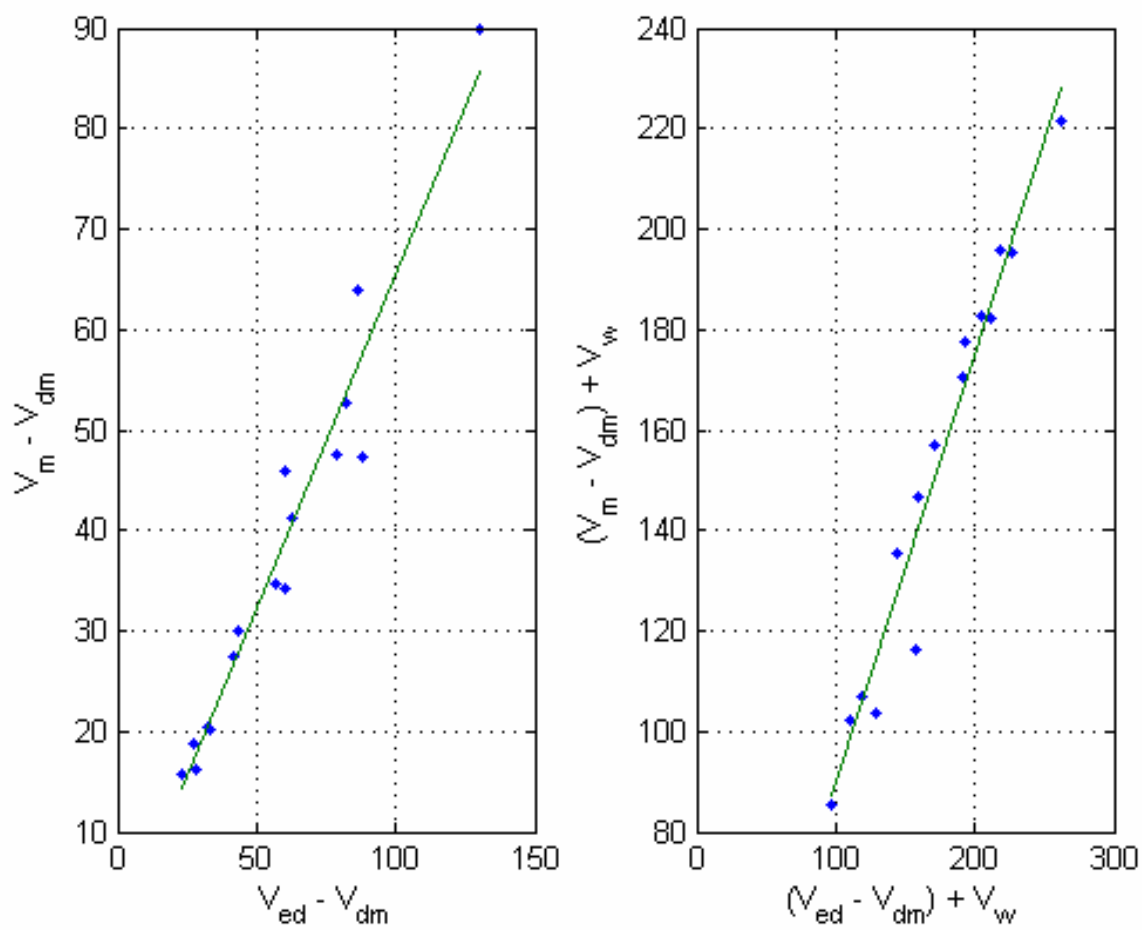

Fig. 8: Left: relationship between $y=\left(V_{m}-V_{d m}\right)$ and $x=\left(V_{e d}-V_{d m}\right)$; least squares fit gives $y=0.6683 x-1.1583$, correlation coefficient $r=0.9762$. Right: relationship between $y=\left(V_{m}-V_{d m}+V_{\omega}\right)$ and $x=\left(V_{e d}-V_{d m}+V_{\omega}\right)$; least squares fit gives $y=0.8558 x+3.9269$, correlation coefficient $r=0.9813$. Experimental data are taken from Burns et al [32] and Shoucri [33]. 